DIE KIRCHEN DER WELT . BAND II

DIE BAPTISTEN 


\section{DIE KIRCHEN DER WELT \\ BAND II}

Herausgeber

D. HANS HEINRICH HARMS

D. FERDINAND SIGG

D. HANS HEINRICH WOLF

Gesamtredaktion: Dr. Gotthold Müller 


\title{
DIE BAPTISTEN
}

\author{
Herausgegeben von
}

J. D. HUGHEY

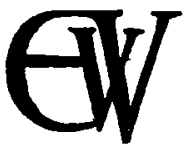

EVANGELISCHES VERLAGSWERK STUTTGART 
Die Übersetzung der im Original fremdsprachlichen Beiträge haben besorgt: Dr. Johannes Arndt die Kapitel I, II, III, VII, IX, X, XI, XII, XIII, XV, XVII, XVIII, Conrad Ihlow Kapitel XVI, Lilian Wieske Kapitel XVI. 